\title{
Análisis del componente rápido de la cinética de recuperación del consumo de oxigeno tras un programa HIIT de 10 dias en un grupo de obesos
}

\author{
Analysis of the fast component of the oxygen uptake kinetics \\ after a 10 day long HIIT program in a group of obese men
}

Alexis Espinoza-Salinas ${ }^{1,2}$; Giovanny Arenas-Sánchez²; Byron Silva-Huenopil ${ }^{1}$; Sebastián Osorio-Marambio ${ }^{1}$; Carla Firinguetti-Balocchi²; Edson Zafra-Santos ${ }^{1}$

Forma de citar: Espinoza-Salinas A, Arenas Sánchez G, Silva Huenopil B, Osorio Marambio S, Firinguetti Balocchi C, Zafra Santos E. Análisis del componente rápido de la cinética de recuperación del consumo de oxígeno tras un programa HIIT de 10 días en un grupo de obesos. Rev Univ Ind Santander Salud. 2018; 50(1): 7-17. doi: http://dx.doi.org/10.18273/revsal.v50n1-2018001 @) (1)

\section{Resumen}

Introducción: El sujeto obeso evidencia una disminución de la capacidad funcional (CF), que entre otros factores comprende una cinética del consumo de oxígeno $\left(\mathrm{VO}_{2}\right)$ más lenta, asociada a una posible relación entre el tejido adiposo y una desregulación crónica del sistema nervioso autónomo, tanto en condiciones de reposo como de ejercicio. Objetivo: Analizar el comportamiento del tau rápido de la cinética off del consumo de oxígeno, luego de la aplicación de un protocolo de ejercicio interválico de alta intensidad y corta duración (HIIT) en un grupo de obesos. Metodología: Se diseñó un estudio comparativo, experimental y longitudinal de corto plazo en cinco sujetos obesos seleccionados por un muestreo no probabilístico intencionado. Se valoraron las siguientes variables: peso, talla, índice de masa corporal (IMC), perímetro de cintura, cinética del consumo de oxígeno y aquellas dependientes del procedimiento. Los datos de $\mathrm{VO}_{2} \mathrm{v} / \mathrm{s}$ tiempo se ingresaron al Software Graph Pad Prism 6, (Graph Pad Prism versión 6.01 para Windows ${ }^{\circledR}$ ) donde se depuraron promediando intervalos de 10 segundos y fueron ajustados a un modelo de función bi-exponencial. Posteriormente se realizó un análisis estadístico descriptivo por medio del Software SigmaPlot 12.5 para Windows ${ }^{\circledR}$ usando las siguientes estadísticas de resumen: media, desviación estándar y rango. Resultados: La edad media fue 25,13 \pm 0,62 años. La mayor variación al analizar los resultados en ambas fases del estudio (pre-intervención: 43,09 \pm 7,78 s; post-intervención: $26,85 \pm 3,78 \mathrm{~s}$ ) se encontró en el tau rápido del $\mathrm{VO}_{2}(\mathrm{p}=0,034)$. Conclusiones: El entrenamiento de alta intensidad y corta duración realizado en un período de 10 días logra un efecto positivo sobre el componente rápido de la cinética off del $\mathrm{VO}_{2}$ para la población estudiada, mejorando así el tiempo de recuperación posterior al esfuerzo físico.

Palabras clave: Entrenamiento interválico de alta intensidad; Obesidad; Consumo de oxígeno; Alteración autonómica; Tejido adiposo.

1. Universidad Santo Tomás. Santiago, Chile.

2. Centro de Ejercicio Adaptado, CEA- YMCA. Santiago, Chile.

Correspondencia: Alexis Espinoza-Salinas, Dirección: Ejército 146, Santiago, Chile, correo electrónico: alexisespinozasa@santotomas.cl. Teléfono: +56224717674 . 


\begin{abstract}
Introduction: Obese subjects exhibit a decreased functional capacity (FC) which, among other factors, entails a slower oxygen uptake kinetics associated to a pathological relationship between the adipose tissue and a chronicallyderegulated autonomic nervous system, both in resting and exercise conditions. Objective: To analyze the fasttau behavior of the oxygen consumption off-kinetics, after applying a high intensity and short duration intervallic exercise protocol (HIIT) in a group of obese men. Methodology: An exploratory, comparative, experimental and longitudinal short-term study of 5 obese subjects, selected by intentional non-probabilistic sampling, was designed. Weight, height, body mass index (BMI), waist circumference, kinetics of oxygen consumption and variables related to the procedure were assessed. Data from $\mathrm{VO}_{2}$ v/s time were compiled with Graph Pad Prism v. 6.01 by averaging 10-second intervals and fitted as a bi-exponential model. Subsequently, a descriptive statistical analysis was performed using SigmaPlot v. 12.5, calculating means, standard deviations and ranks. Results: Mean age was $25.13 \pm 0.62$ years. Highest variation when analyzing the results, in both phases of the study (pre-intervention: $43.09 \pm 7.78 \mathrm{~s}$; post-intervention: $26.85 \pm 3.78 \mathrm{~s}$ ), was found in the fast $\mathrm{VO}_{2} \tan (\mathrm{p}=0.034)$. Conclusion: The highintensity, short-duration training performed over a 10-day period, achieves a positive effect on the rapid kinetic component of $\mathrm{VO}_{2}$ for the studied population, improving recovery time after physical exertion.
\end{abstract}

Keywords: High-Intensity Interval Training; Obesity; Oxygen consumption; Autonomic disease; Adipose tissue.

\section{Introducción}

La obesidad constituye un creciente problema de salud, el cual se ha acelerado debido a sus comorbilidades ${ }^{1,2}$. Ésta se define como una enfermedad crónica de origen multifactorial, asociada con un aumento en la acumulación de grasa corporal, vinculada a disminución de la capacidad funcional (CF), capacidad oxidativa muscular deteriorada y cinética del consumo de oxígeno $\left(\mathrm{VO}_{2}\right)$ más lenta, lo que provoca una disminución de la tolerancia al esfuerzo ${ }^{3,4}$.

Entre los mecanismos que explicarían la disminución de la CF en el paciente obeso está la modificación de su patrón respiratorio. Este cambio respondería al desplazamiento craneal del diafragma debido a un aumento de la presión abdominal, que conduce a una disminución exponencial en el volumen de reserva espiratorio y el volumen pulmonar espiratorio final (capacidad residual funcional) $^{5}$. Esta modificación en la mecánica del diafragma resulta en una disminución del volumen corriente, concomitante con aumentos en la frecuencia respiratoria y en el espacio muerto. Esto podría responder a un mecanismo compensatorio, no obstante, insuficiente para disminuir la carga mecánica solicitada al sistema toraco-pulmonar y así controlar la disnea durante el esfuerzo ${ }^{6}$. Lo anterior redunda en un aumento de la demanda ventilatoria con mayor $\mathrm{VO}_{2}$ de la musculatura respiratoria, el que se ha descrito hasta tres veces superior al de los sujetos normopeso ${ }^{7}$. Además, los sujetos obesos tanto en reposo como en ejercicio experimentan una mayor demanda de sus músculos inspiratorios, lo que se asocia a condiciones de inactividad física propias del desarrollo de la obesidad, donde se impone una condición de sarcopenia con una mayor pérdida de fibras oxidativas ${ }^{8}$, lo que también contribuiría a la disminución de la CF.

El estudio del $\mathrm{VO}_{2}$ y su regulación son importantes debido a que el metabolismo oxidativo es la principal vía por la cual el organismo humano genera energía para realizar trabajo en casi todas las actividades cotidianas. Los factores tales como: (i) el mayor $\mathrm{VO}_{2}$ alcanzable, (ii) el $\mathrm{VO}_{2}$ requerido para realizar ejercicio submáximo, y (iii) la tasa a la cual el $\mathrm{VO}_{2}$ se eleva durante el tránsito aeróbico-anaeróbico (de una menor a una mayor demanda energética hasta alcanzar el estado estable), influenciarán la tolerancia individual a la actividad física9. En esta línea, el estudio de la cinética del $\mathrm{VO}_{2}$ permite analizar los mecanismos fisiológicos responsables de la respuesta dinámica del $\mathrm{VO}_{2}$ al ejercicio y su subsiguiente recuperación. De su análisis se extrae la cinética del $\mathrm{VO}_{2}$, definida como el estudio de los mecanismos fisiológicos responsables de la respuesta dinámica del $\mathrm{VO}_{2}$ frente al ejercicio físico y su posterior recuperación ${ }^{10,11}$, que según la literatura puede desarticular su estructura en dos (Figura 1) $)^{12,13}$. En primer lugar, se reconoce la transición del $\mathrm{VO}_{2}$ desde el reposo al ejercicio, proceso conocido como cinética on del $\mathrm{VO}_{2}$, entendido comúnmente por un modelo de ajuste trifásico o tri-exponencial caracterizado por una fase I o cardiodinámica, una fase II o principal y una fase III o estado estable ${ }^{14-16}$.

Mientras que la transición inversa del $\mathrm{VO}_{2}$ desde el ejercicio al reposo se identifica como cinética off del $\mathrm{VO}_{2}$ o cinética de recuperación, definida como el proceso comprendido entre el $\mathrm{VO}_{2}$ alcanzado al final del ejercicio $\left(\mathrm{EEVO}_{2}\right)$ y su desaceleración de retorno al reposo. 


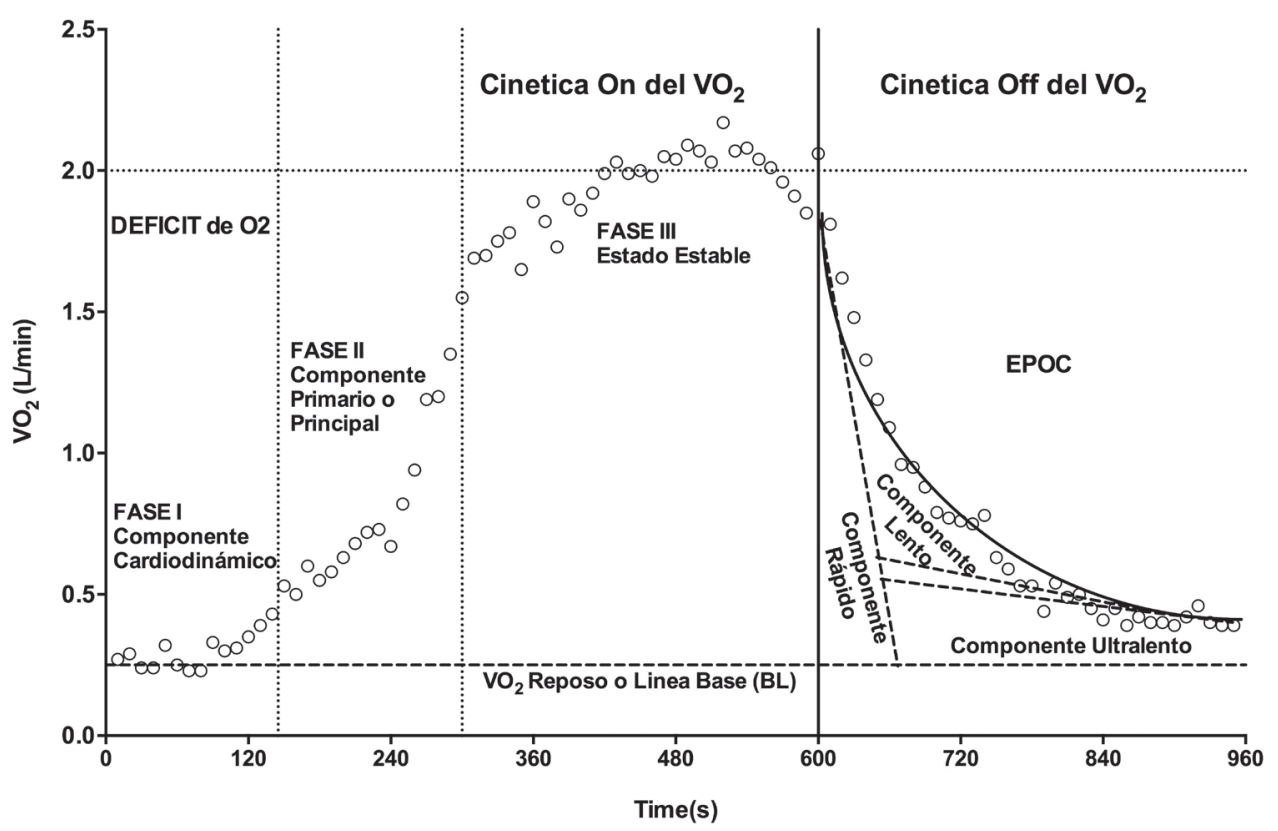

Figura 1. Modelo descriptivo de tres fases para la cinética on y off del consumo de oxígeno, y el proceso de recuperación post ejercicio, en base a un ejercicio de carga constante.

De este proceso se extrae un concepto que se remonta a la década de 1920, donde Hill, et al. ${ }^{17,18}$ formularon una hipótesis conocida como "deuda de oxígeno", teoría que vinculaba el metabolismo del ácido láctico con el exceso de $\mathrm{VO}_{2}$ producido posterior a la realización de ejercicio.

Pero finalmente fueron Gaesser, et at. ${ }^{19}$ en el año 1984 quienes acuñaron el término exceso de consumo de oxígeno post-ejercicio o EPOC, identificándose según la literatura tres componentes del EPOC: el componente rápido, con una duración de 10 segundos a unos pocos minutos, caracterizado por una vía anaeróbica aláctico o ATP-PC ${ }^{20}$; el componente lento, que puede durar entre algunos minutos a horas dependiendo del desequilibrio en la homeostasis provocado por el ejercicio, representado por una vía anaeróbica láctica o glucolítica ${ }^{21}$ y el componente ultralento, con una duración de 48 horas post-ejercicio, dependiente de una vía aeróbica u oxidativa caracterizada por una alta tasa metabólica ${ }^{22-24}$.

En este contexto, el incremento de la grasa corporal se ha correlacionado con mayor actividad simpática en reposo, con niveles de hasta $50 \%$ superior a los sujetos sanos, y con una menor actividad parasimpática ${ }^{25}$, asociada a la disminución de la sensibilidad barorrefleja, interesantemente se ha determinado que el balance simpático-vagal en reposo en obesos está inversamente relacionado con el $\mathrm{VO}_{2}$, reflejo de la $\mathrm{CF}$ y recuperación del $\mathrm{VO}_{2}{ }^{26}$.

Por otra parte, tradicionalmente los programas de ejercicio en modalidad aeróbica continua y últimamente de fuerza han sido utilizados como los principales métodos de intervención para mejorar los parámetros de la CF y recuperación. La combinación de ambos protocolos de ejercicio es la vía más efectiva para alcanzar resultados beneficiosos ${ }^{27}$.

En este sentido, la poca disponibilidad de tiempo, el alto volumen de trabajo y la búsqueda de efectos a corto plazo, son algunas de las razones por las que los obesos tienen poca adherencia a los protocolos de ejercicio ${ }^{28}$, un protocolo de alta intensidad y corta duración podría favorecer la adherencia a los programas de ejercicio.

Existen experiencias y propuestas orientadas a verificar si un entrenamiento de alta intensidad y corta duración podría provocar mejoras en la CF y la recuperación ${ }^{29}$. El método propuesto consiste en cinco series de cinco segundos y 55 segundos de recuperación $5 \times 5 \times 55^{13}$.

A partir de lo anterior surge la siguiente la hipótesis de trabajo: el entrenamiento de alta intensidad y corta duración mejora el componente rápido de la cinética de recuperación del consumo de oxígeno, de donde se desprende el objetivo del presente manuscrito. 


\section{Metodología}

Diseño: Este estudio presenta un diseño exploratorio, comparativo, experimental y longitudinal de corto plazo en un grupo de individuos, para el cual se analizó el componente rápido de la cinética de recuperación del consumo de oxígeno comparando los datos obtenidos pre y post intervención. Esto último debido a que es un método apropiado para cuantificar las variaciones producidas como consecuencia del protocolo de ejercicio aplicado en el grupo en cuestión. La ventaja de este diseño es la existencia de un punto de referencia inicial de los sujetos en estudio antes del estímulo ${ }^{30}$.

Participantes: Se utilizó una muestra intencionada no probabilística, para la cual se seleccionaron hombres de entre 18 y 30 años con sobrepeso u obesidad, con un índice de masa corporal (IMC) entre 25 y $35\left(\mathrm{~kg} / \mathrm{m}^{2}\right)$. Dentro de los criterios de inclusión se consideró que los sujetos fuesen físicamente inactivos (de acuerdo al Cuestionario Internacional de Actividad Física (IPAQ), puntaje $I P A Q<600$ minutos/semana), sin patologías asociadas, con acceso a una bicicleta estática una vez al día y dispositivos de telefonía móvil. Los sujetos que hubieran realizado ejercicio físico vigoroso durante las últimas dos semanas, no sedentarios, con hábito tabáquico, con enfermedades asociadas o que tuvieran prohibición o limitación médica para realizar ejercicio fueron excluidos del estudio. Los criterios de eliminación fueron: el no cumplimiento del protocolo de evaluación inicial o final, angina, disnea severa, diplopía o náuseas durante el protocolo de evaluación Astrand \& Rhyming, el no cumplimiento de la realización del protocolo de ejercicio físico autoadministrado, el haber realizado algún protocolo de ejercicio físico y/o entrenamiento en forma adicional al indicado para este estudio o presentar alguna enfermedad durante el período de entrenamiento que impidiera seguir con el mismo.
De un total de 14 sujetos que se presentaron voluntariamente para participar en el estudio, cuatro evidenciaron criterios de exclusión y otros cuatro cumplieron con alguno de los criterios de eliminación, por lo que la muestra se redujo inicialmente a un tamaño de seis sujetos, quienes firmaron un consentimiento informado, aceptando ser evaluados y comprometiéndose a entregar los datos solicitados con la mayor legitimidad posible. Cabe destacar que, debido a un error procedimental, los datos de uno de los sujetos fueron eliminados del estudio al no cumplir con los requerimientos para su análisis, razón por la que la muestra considerada para determinar los parámetros relacionados con la cinética off del $\mathrm{VO}_{2}$ fue de cinco sujetos en total (edad $=25,13 \pm 0,62$ años, $\mathrm{IMC}=30,83$ $\pm 1,39 \mathrm{~kg} / \mathrm{m}^{2}$ ). Sus datos antropométricos pre y post intervención se visualizan en la Tabla 1 y el flujograma respectivo en la Figura 2.

Tabla 1. Valores promedios y desviaciones estándar de las características antropométricas de los individuos en ambas fases del estudio.

\begin{tabular}{ccc}
\hline & $\begin{array}{c}\text { Pre - intervención } \\
\text { Media } \pm \text { PS } \\
\text { (rango) }\end{array}$ & $\begin{array}{c}\text { Post - intervención } \\
\text { Media } \pm \text { DS } \\
\text { (rango) }\end{array}$ \\
\hline n=5 & 5 & 5 \\
Hombres & & \\
Edad & $25,13 \pm 0,62$ & - \\
(años) & $(19-28)$ & \\
Talla & $173,04 \pm 3,02$ & - \\
(cm) & $(167-188)$ & \\
Peso & $95,20 \pm 7,67$ & $96,04 \pm 7,62$ \\
(kg) & $(69,20-119)$ & $(71,50-120)$ \\
IMC & $30,83 \pm 1,39$ & $31,23 \pm 1,33$ \\
(kg/m $\left.{ }^{2}\right)$ & $(25-35,67)$ & $(26-36)$ \\
Perímetro cintura & $105,38 \pm 2,97$ & $102,49 \pm 3,46$ \\
(cm) & $(95-114)$ & $(89-111)$ \\
\hline
\end{tabular}

DS: Desviación estándar; cm: Centímetros; kg: Kilogramos; m: Metros; IMC: Índice de masa corporal.

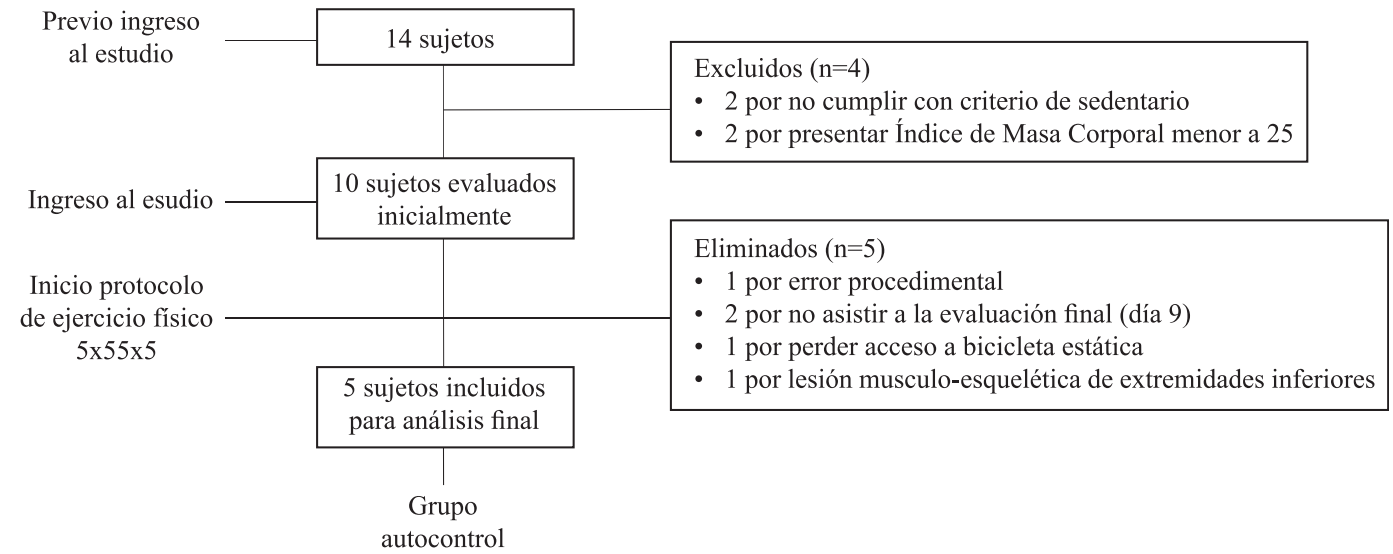

Figura 2. Flujograma descriptivo de la determinación de la muestra utilizada en la investigación. 
Procedimiento: Previo a la medición inicial, los participantes firmaron un consentimiento informado, mediante el cual se les puso al tanto sobre los riesgos, beneficios e instrucciones que debían seguir durante la realización del estudio, solicitando su autorización para el uso de los datos que se obtuvieran a partir de las mediciones.

Día 1: Se llevó a cabo una evaluación inicial para obtener datos personales, antropométricos y de ergoespirometría. Se registró la talla en centímetros (cm) - utilizando un tallímetro (ADE, Arquimed) - y el peso en kilogramos $(\mathrm{kg})$, para lo que se le solicitó a cada sujeto estar descalzo y en ropa interior. A continuación, el individuo se vistió con indumentaria deportiva y se le explicó tanto la Escala de Borg como el procedimiento de ergoespirometría. El sujeto se ubica en el cicloergómetro (Monark, modelo Ergomedic 874E) regulando previamente la altura del asiento a nivel de la cadera y ajustando la máscara (Azul, Medium) de manera que no exista fuga de aire. Finalmente, se ingresan los datos del participante al software Metalyzer 3B-R2 del ergoespirómetro, dando inicio a la prueba.

La evaluación ergoespirométrica tuvo una duración de 22 minutos y se realizó de la siguiente forma: se le solicitó al sujeto permanecer sobre el cicloergómetro en reposo absoluto, respirando calmado durante seis minutos para obtener los datos de $\mathrm{VO}_{2}$ reposo y la percepción de esfuerzo a través de la escala de Borg. Luego se llevó a cabo un calentamiento de dos minutos, en los que el sujeto debía pedalear a 50 revoluciones/ pedal/minuto (rpm) sin carga, obteniéndose datos respiración a respiración y la percepción del esfuerzo durante los últimos 15 segundos. Posteriormente se aplicó el protocolo submáximo de Astrand \& Rhyming modificado en cicloergómetro durante seis minutos, tiempo en el que se debía mantener el pedaleo a $50 \mathrm{rpm}$ con una carga ajustada para sujetos desentrenados (50 a 100 Watts), obteniendo la percepción de esfuerzo en los últimos 15 segundos de cada minuto y la frecuencia cardíaca (FC) a través de un cardiotacómetro (Polar RS800 CX). Por último, se ejecutó una fase de retorno a la calma durante dos minutos, retirando gradualmente la carga, pero solicitando al sujeto que mantuviera el pedaleo a $50 \mathrm{rpm}$, obteniendo la percepción del esfuerzo durante los últimos 15 segundos. Una vez realizado lo anterior, el sujeto debía descender del cicloergómetro y posicionarse en sedente en una silla con respaldo y apoyabrazos, de modo que pudiera respirar en forma tranquila durante seis minutos, obteniéndose datos respiración a respiración y la percepción del esfuerzo al término de dicho tiempo.
Finalizada la medición inicial, se indicaron las instrucciones que debían seguir los participantes, explicando el protocolo de ejercicio $5 \times 55 \times 5^{13}$, el cual debían realizar una vez al día, durante los 10 días posteriores a la evaluación, utilizando la bicicleta estática a la que refirieron tener acceso (sin carga).

Se llevó un control de la aplicación del protocolo por vía telefonía móvil usando la aplicación WhatsApp, y se les entregó una planilla en la que debían registrar su percepción del esfuerzo, según la escala de Borg con el propósito de controlar posibles lesiones. Durante los 10 días de intervención, se les indicó no realizar ningún otro tipo de entrenamiento físico, restringir el consumo de alcohol, cigarrillos y seguir su dieta en forma normal.

Día 2 al 11: Los sujetos llevaron a cabo el protocolo $5 \times 55 \times 5^{13}$ en su domicilio. Para esto se les recordó diariamente la realización del mismo y las instrucciones señaladas vía WhatsApp.

Día 12: Se aplicó la misma evaluación del día 1, registrando nuevamente a los participantes, para obtener los datos previos y posteriores a la intervención.

Cinética del consumo de oxígeno: Los procesos fisiológicos responsables de las variaciones en el $\mathrm{VO}_{2}$ en situación de ejercicio y su posterior recuperación dan lugar a la cinética del $\mathrm{VO}_{2}{ }^{31,32}$. En particular, para los fines de este estudio se abordó la cinética de recuperación y se registraron las variables asociadas: tau fast (constante tiempo de la fase rápida), tau slow (constante tiempo de la fase lenta), $\mathrm{VO}_{2}$ reposo (consumo máximo de oxígeno en reposo), $\mathrm{EEVO}_{2}\left(\mathrm{VO}_{2}\right.$ alcanzado al final del ejercicio), amplitud de la transición off (diferencia entre el $\mathrm{EEVO}_{2}$ y el $\mathrm{VO}_{2}$ reposo) y cinética off del $\mathrm{VO}_{2}$ (entendida como el $\mathrm{VO}_{2}$ promedio de las fases rápida y lenta), aunque el análisis se centró principalmente en el componente rápido. Los datos de $\mathrm{VO}_{2}$ y tiempo para la construcción de las curvas de cinética off de consumo de oxígeno se obtuvieron respiración a respiración mediante ergoespirometría, siguiendo el protocolo de Astrand \& Ryhming (día 1 y día 12) puesto que al ser submáximo disminuye la probabilidad de interrumpirlo por fatiga muscular local o factores ortopédicos, más que por limitaciones cardiopulmonares ${ }^{33}$. Si bien los sujetos no presentaban enfermedades asociadas, este protocolo evitaba los riesgos cardiovasculares asociados al desarrollo de protocolos maximales en obesos $^{34}$. Además, otro criterio por el que se usó es que para mantener la simetría entre el tau on-off ${ }^{\beta 1,35}$ el ejercicio debe ser realizado en el dominio de intensidad moderada, evitando la aparición del componente lento y las asimetrías dinámicas entre la cinética on-off, 
resultando en un mejor tratamiento de los datos por medio del ajuste bi-exponencial de la curva ${ }^{36-38}$.

Protocolo de ejercicio 5x55x5: Conceptualmente se define como un protocolo de ejercicio de transición ejercicio-reposo de alta intensidad que tiene como objetivo modificar el balance autonómico, aumentando la activación vagal y favoreciendo la retirada simpática. Desde el punto de vista operacional nos referimos específicamente al protocolo de Duarte, et al. ${ }^{13}$, que en este caso se llevó a cabo en bicicleta estática, siendo ajustada ergonómicamente por cada individuo, quienes recibieron previamente instrucciones para su ejecución. Este protocolo, que fue ejecutado una vez al día durante los 10 días de intervención (día 2 al 11) consta de dos fases: fase 1 - el individuo permanece sentado durante 55 segundos sin pedalear (fase pasiva); fase 2 - pedaleo a la máxima velocidad posible ( $\sin$ carga) durante cinco segundos (fase activa). Este ciclo debe repetirse cinco veces, con una duración total de cinco minutos. La elección de este protocolo de entrenamiento, que fue autoadministrado por los participantes, se basó en su naturaleza intermitente, su corta duración y fácil aplicación.

Análisis estadístico: Los datos de $\mathrm{VO}_{2} \mathrm{v} / \mathrm{s}$ tiempo que fueron obtenidos los días 1 y 12 se ingresaron al Software Graph Pad Prism 6, (Graph Pad Prism versión 6.01 para Windows $\left.{ }^{\circledR}\right)$ donde se depuraron promediando intervalos de 10 segundos y fueron ajustados a un modelo de función bi-exponencial para la elaboración del gráfico ilustrativo de la cinética off del $\mathrm{VO}_{2}$ y sus componentes. Resultó esencial la incorporación de un modelo de tipo bi-exponencial (two phase decay) de regresión no lineal ya que permite dividir la curva de recuperación en un componente rápido y otro lento, además de proporcionar la extracción de parámetros relevantes, como las constantes de tiempo (tau) y velocidad, la amplitud y el $\mathrm{VO}_{2}$ reposo $^{36,39}$. Con esto se logra examinar los cambios inmediatamente posteriores al ejercicio, separando la fase lenta sobre la cual el protocolo autoadministrado $5 \times 55 \times 5$ ejercería efectos menos visibles.

Posteriormente se realizó un análisis estadístico descriptivo por medio del Software SigmaPlot 12.5 para Windows ${ }^{\circledR}$ usando los recursos estadísticos de la media, desviación estándar y rango. De acuerdo a los resultados de la prueba de Shapiro-Wilk los datos siguen una distribución normal.

Los valores previos y posteriores a la realización del protocolo de ejercicio físico $5 \times 55 \times 5^{13}$ se analizaron mediante prueba t de Student pareada para identificar la existencia de significancia estadística entre cada 12 variable. El criterio de significancia estadística utilizada fue un valor $\mathrm{p}<0,05$, para un intervalo de confianza de $95 \%$.

\section{Resultados}

Las características antropométricas, sus valores promedios y desviaciones estándar se observan en la Tabla 1. El mayor cambio lo presentó el perímetro de cintura (pre-intervención: 105,38 $\pm 2,97 \mathrm{~cm}$; postintervención: $102,49 \pm 3,46 \mathrm{~cm}$ ), no significativo. Las variables relacionadas a la cinética off del $\mathrm{VO}_{2}$ se observan en la Tabla 2. Al analizar los resultados en ambas fases del estudio (pre-intervención: 43,09 \pm 7,78 s; post-intervención: $26,85 \pm 3,78$ s) se encontró un valor $(\mathrm{p}=0,034)$ en el tau rápido del $\mathrm{VO}_{2}$. Al comparar los valores del tau lento, existe un aumento en los valores medios luego de la intervención (preintervención: $90,76 \pm 7,83 \mathrm{~s}$; post-intervención: 95,9 $\pm 18,2 \mathrm{~s})$, sin embargo, no presentan diferencias significativas $(\mathrm{p}=0,667)$. Tampoco lo hubo para el $\mathrm{VO}_{2}$ al final del ejercicio $\left(\mathrm{EEVO}_{2}\right)$ posterior al entrenamiento $(\mathrm{p}=0,277)$, a pesar del aumento del valor medio (preintervención: $2,247 \pm 0,267 \mathrm{~L} / \mathrm{min}$; post-intervención: $2,486 \pm 0,267 \mathrm{~L} / \mathrm{min})$. La gráfica representativa de la cinética off del $\mathrm{VO}_{2}$ obtenida en las evaluaciones previo (día 1) y posterior (día 12) a la realización del protocolo $5 \times 55 \times 5$ para un sujeto se observa en la Figura 3.

Tabla 2. Cinética off del $\mathrm{VO}_{2}$ en ambas fases del estudio.

\begin{tabular}{|c|c|c|c|}
\hline & $\begin{array}{c}\text { Pre - intervención } \\
\text { Media } \pm \text { DS } \\
\text { (rango) }\end{array}$ & $\begin{array}{c}\text { Post - intervención } \\
\text { Media } \pm \text { DS } \\
\text { (rango) }\end{array}$ & $\begin{array}{c}\text { Valor } \\
\mathbf{P}\end{array}$ \\
\hline $\begin{array}{c}n=5 \\
\text { Hombres }\end{array}$ & 5 & 5 & \\
\hline $\begin{array}{c}\text { Tau } \\
\text { Rápido } \\
\mathrm{VO}_{2}(\mathbf{s})\end{array}$ & $\begin{array}{c}43,09 \pm 7,78 \\
(18,94-62,10)\end{array}$ & $\begin{array}{c}26,85 \pm 3,78 \\
(20,02-40,46)\end{array}$ & $0,034 *$ \\
\hline $\begin{array}{c}\text { Tau } \\
\text { Lento } \\
\text { VO2 (s) }\end{array}$ & $\begin{array}{c}90,76 \pm 7,83 \\
(78,48-121,2)\end{array}$ & $\begin{array}{c}95,9 \pm 18,2 \\
(50,06-161,7)\end{array}$ & 0,667 \\
\hline $\begin{array}{l}\text { EEVO }_{2} \\
(\mathrm{~L} / \mathrm{min})\end{array}$ & $\begin{array}{c}2,247 \pm 0,267 \\
(1,460-2,699)\end{array}$ & $\begin{array}{c}2,486 \pm 0,267 \\
(1,695-2,996)\end{array}$ & 0,277 \\
\hline $\begin{array}{c}\mathrm{VO}_{2} \text { Basal } \\
(\mathrm{L} / \mathrm{min})\end{array}$ & $\begin{array}{c}0,350 \pm 0,024 \\
(0,284-0,425)\end{array}$ & $\begin{array}{c}0,380 \pm 0,041 \\
(0,318-0,542)\end{array}$ & 0,333 \\
\hline $\begin{array}{l}\text { Amplitud } \\
\mathrm{VO}_{2} \\
(\mathrm{~L} / \mathrm{min})\end{array}$ & $\begin{array}{c}1,896 \pm 0,274 \\
(1,081-2,381)\end{array}$ & $\begin{array}{c}2,105 \pm 0,196 \\
(1,373-2,450)\end{array}$ & 0,344 \\
\hline $\begin{array}{c}\mathrm{VO}_{2} \\
\text { Cinética Off } \\
(\mathrm{L} / \mathrm{min})\end{array}$ & $\begin{array}{c}0,646 \pm 0,030 \\
(0,555-0,733)\end{array}$ & $\begin{array}{c}0,708 \pm 0,065 \\
(0,501-0,853)\end{array}$ & 0,395 \\
\hline
\end{tabular}

DS: Desviación estándar; $\mathrm{VO}_{2}$ : Consumo de Oxígeno; $\mathrm{EEVO}_{2}$ : Consumo de Oxígeno al final del ejercicio. (*) Significancia estadística valor $\mathrm{p}<0,05$. 


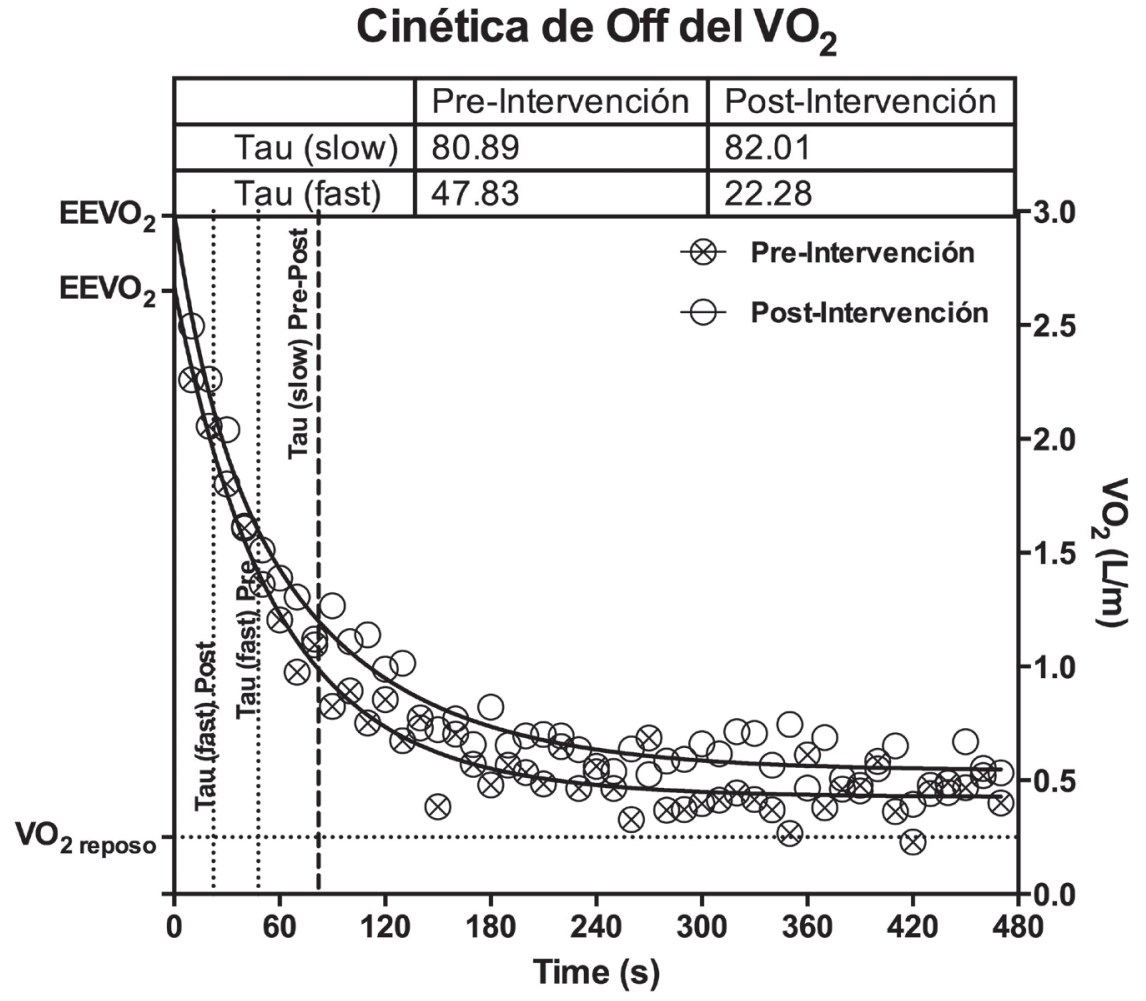

Figura 3. Gráfica representativa de un sujeto, que muestra el comportamiento de la curva de la cinética off del $\mathrm{VO}_{2}$, previo y posterior a la realización del protocolo $5 \times 55 \times 5$ durante 10 días.

\section{Discusión}

El objetivo del presente estudio fue analizar el comportamiento del tau rápido de la cinética off del $\mathrm{VO}_{2}$, posterior a la aplicación del protocolo $5 \times 55 \times 5$ en sujetos con sobrepeso y obesidad ${ }^{13}$. Para esto, se valoraron parámetros de la cinética off del $\mathrm{VO}_{2}$, previo y posterior al protocolo mediante una ergoespirometría que evidenció una disminución del tau rápido, posterior a la realización del protocolo $5 \times 55 \times 5$, sobre la cinética de recuperación para este tipo de población ${ }^{40}$. A pesar de existir poca evidencia dirigida hacia la cinética de recuperación ${ }^{41,42}$ y de ser definido este estudio como exploratorio, se hallaron resultados estadísticamente significativos ( $\mathrm{p}=0,034$; Pre-intervención: 43,09 s; Post-intervención: 26,85 s) sobre la variable de interés. Pese a obtener cambios significativos en el valor postintervención del componente rápido, específicamente en el tau, el resto de los parámetros no logró un cambio estadísticamente significativo. Sin embargo, se observa una tendencia hacia valores esperables según la literatura, correspondiente a un aumento en el tau lento (s), $\mathrm{EEVO}_{2}(\mathrm{~L} / \mathrm{min}), \mathrm{VO}_{2}$ reposo (L/min), Amplitud (L/min) y $\mathrm{VO}_{2}$ cinética off $(\mathrm{L} / \mathrm{min})^{36,39,40,42,43}$. Tendencia que tendría como respuesta, el corto período de desarrollo del entrenamiento, no así su volumen, puesto que los resultados muestran un cambio existente en la cinética de recuperación. Queda abierta la posibilidad de replicar estudios hacia un período de entrenamiento más extenso con este tipo de protocolos, puesto que el pico de cambio más frecuentemente evidenciado en cinética del $\mathrm{VO}_{2}$ se encuentra cercano a la cuarta semana de entrenamiento ${ }^{42,43}$, a raíz de la adaptación al entrenamiento descrita en numerosa bibliografía ${ }^{20,44}$.

A diferencia de lo señalado en los párrafos anteriores, la metodología utilizada en el presente estudio se orientó hacia el conocimiento de respuestas y adaptaciones iniciales al entrenamiento, para el aporte en aspectos menos estudiados de la cinética del $\mathrm{VO}_{2}$, como es el componente rápido, tanto en lo relacionado a la cinética on y off, además de sus transiciones.

Un segundo factor importante se asocia a la población en estudio, considerando a la obesidad y el sobrepeso como un condicionante a la respuesta encontrada ${ }^{43}$, puesto que la asociación de esta patología a factores cardiometabólicos como la disfunción autonómica, hipertensión arterial y aumento de grasa corporal, sumado a elementos psicosociales como la autoestima y los hábitos ${ }^{45}$, actúan integrándose en una respuesta 
atenuada frente al ejercicio, dificultando su rendimiento y enlenteciendo los cambios en comparación a sujetos sanos.

En particular, la diferencia que presenta el componente lento y rápido posterior a la intervención, responde directamente a las rutas metabólicas utilizadas según la demanda impuesta por el ejercicio, evidenciándose una relación principalmente asociada a la intensidad y no necesariamente al volumen de entrenamiento ${ }^{43,46}$.

Otro factor importante para la comprensión de la cinética del $\mathrm{VO}_{2}$, es la construcción de modelos matemáticos que permitan disociar los componentes de la curva, para así lograr un estudio acabado de cada uno de los elementos que constituyen la respuesta dinámica del $\mathrm{VO}_{2}$ frente al ejercicio y su posterior recuperación ${ }^{37}$.

Diversos factores y mecanismos regulan la respuesta del $\mathrm{VO}_{2}$ frente al ejercicio; centrales y periféricos, los que hace muchas décadas atrás comenzaron a ser cuestionados y que aún siguen en discusión ${ }^{31}$.

Dentro de los factores centrales, el sistema pulmonar y cardiovascular son los responsables de la cinética del $\mathrm{VO}_{2}$ durante el ejercicio. El sistema pulmonar modula la respuesta a través una de hiperepnea generada a nivel de quimiorreceptores periféricos, para facilitar la remoción de $\mathrm{CO}_{2}$ y no alterar la presión parcial de oxígeno arterial y el descenso brusco del pH. El sistema cardiovascular responde al ejercicio, con un aumento de su FC, volumen eyectivo y gasto cardíaco, con la finalidad de satisfacer la demanda de $\mathrm{O}_{2}$ de los músculos utilizados. Los cambios súbitos de FC en las transiciones reposoejercicio-reposo de la fase rápida de la cinética del $\mathrm{VO}_{2}$ tienen relación con mecanismos simpático-vagales, que en ejercicios de moderada intensidad responden a una retirada vagal, y en ejercicios de alta intensidad, a una sumación de la retirada vagal y estimulación simpática ${ }^{31}$.

Con respecto a lo último, las adaptaciones al protocolo de ejercicio aplicado en este estudio, podrían responder al descenso en el tiempo de la cinética off. Los hallazgos del estudio de Duarte, et al. ${ }^{13}$, señalan que luego de ocho semanas de entrenamiento $5 \times 55 \times 5$, existen cambios en el tono vagal cardíaco en sujetos adultos, lo que se traduce en predominancia de modulación parasimpática, que para sujetos con alteraciones cardíacas o metabólicas es importante. Esto concuerda con los hallazgos, puesto que al modular la función autonómica a través del entrenamiento $5 \times 55 \times 5$, los sujetos, podrían tolerar mejor el ejercicio físico y recuperarse más rápido, con base en los cambios en el componente central cardíaco que responde frente a la recuperación de la fase rápida de la cinética del $\mathrm{VO}_{2}{ }^{16,31}$, sin embargo, se debe interpretar cuidadosamente estos resultados, ya que podrían existir otras variables que afecten los resultados, como adaptaciones a nivel periférico.

Los mecanismos periféricos tienen que ver con la utilización del $\mathrm{O}_{2}$ entregado a nivel muscular, flujo sanguíneo muscular, enzimas mitocondriales, densidad capilar y el sistema microvascular muscular, son los factores periféricos que intervienen en la cinética del $\mathrm{VO}_{2}$ frente al ejercicio ${ }^{16,31,47}$.

Con respecto a ésto, McNarry, et al ${ }^{48}$ investigaron la influencia de un HIIT sobre la cinética del $\mathrm{VO}_{2}$ en obesos adolescentes, y los resultados indicaron diferencias significativas en el tau de la cinética on luego del periodo de intervención, lo cual sustenta la evidencia hallada, considerando que los mecanismos responsables del aumento de la cinética rápida son similares en ambas fases on-off. Además, al comparar la relación entre el $\mathrm{VO}_{2}$ pico y el tau on, considerados ambos como marcadores de fitness aeróbico, no se encontraron mejoras significativas en el $\mathrm{VO}_{2}$ pico, sugiriendo que aquello se podría deber a los factores predominantes para cada variable; factores intrínsecos musculares (relacionados a la cinética) y factores cardiovasculares centrales (relacionados al $\mathrm{VO}_{2}$ pico). De ello se infiere que el entrenamiento interválico aplicado tuvo más influencia en factores periféricos que en centrales.

Otro estudio sobre factores periféricos (cambios celulares musculares) señala que luego de tres horas de realizado un HIIT, se produce un aumento en la fracción de mRNA de PGC-1 $\alpha$, la cual es considerada clave en la biogénesis mitocondrial ${ }^{49}$. Actuando como un coactivador de receptores nucleares que inducen una cascada que estimula la transcripción mitocondrial.

El aumento en la transcripción mitocondrial favorece múltiples procesos, como la fosforilación oxidativa y oxidación lipídica, lo que a su vez, favorecería la resíntesis de fosfágenos de alta energía, proceso que está relacionado con el componente rápido de la cinética de recuperación, por lo que su mejora, favorece la recuperación posterior a la realización de ejercicio $^{22,29,49,50}$.

Todo aquello podría relacionar los cambios significativos hallados en el tau rápido con el entrenamiento HIIT, considerándose a este como un promotor de la biogénesis mitocondrial y capacidad oxidativa 
muscular en sujetos con sobrepeso y obesidad, los que tienen directa relación con los mecanismos periféricos de regulación del $\mathrm{VO}_{2}{ }^{29,47,49}$.

Por otra parte, la obesidad se relaciona con desacondicionamiento físico, el cual limita la funcionalidad, el desempeño motor y la tolerancia al ejercicio físico, experimentando una sensación habitual de cansancio, lo que repercute en su calidad de vida. La literatura sugiere, que son tanto factores musculoesqueléticos como cardiovasculares los causantes de lo anteriormente señalado. Con respecto a los factores musculoesqueléticos, Salvadego, et al. ${ }^{34}$, mencionan un aumento en la proporción de fibras musculares rápidas, las que son menos resistentes a la fatiga, comparadas a las fibras tipo I. Además, poseen un menor contenido y capacidad mitocondrial, lo que tiene directa relación con la pobre tolerancia al ejercicio en los sujetos obesos. Esto último, se manifiesta en la respuesta dinámica pulmonar del $\mathrm{VO}_{2}$, en donde se reconoce una cinética menor comparado con sujetos sanos o sujetos entrenados ${ }^{43,48}$. La cinética del $\mathrm{VO}_{2}$ refleja la tasa de cambio que se experimenta al variar la demanda y requerimientos metabólicos, de acuerdo a la actividad física realizada, lo que para los sujetos obesos ocurre frecuentemente en actividades de la vida diaria. En estas situaciones de transiciones entre reposoejercicio-reposo (cinética on-off), una cinética lenta, genera un mayor déficit de oxígeno, determinando la necesidad de mayor fosforilación a nivel de sustrato lo que está inversamente relacionado con la tolerancia al ejercicio y estabilidad metabólica ${ }^{34}$. Como se ha señalado anteriormente, el entrenamiento interválico de alta intensidad, promueve mejoras significativas a nivel muscular y cardiovascular, lo que permitiría mejorar la cinética off para promover una recuperación precoz luego de realizar alguna actividad física, siendo el protocolo $5 \times 55 \times 5$ una alternativa para aquello de acuerdo a los resultados de nuestro estudio.

\section{Limitaciones del estudio}

Se encuentra la autoadministración del protocolo, la cual fue controlada por medio de "WhatsApp" y un documento de compromiso, solicitando a los sujetos la legitimidad frente a los datos entregados, puesto que conforman parte de un material de investigación. Sin embargo, no es posible afirmar con certeza que cada sujeto completó el entrenamiento señalado, pero sí manifestaron sentirse cómodos con él y en algunos casos señalaron querer continuarlo. Otra limitante es el pequeño tamaño de la muestra, y a pesar de que la distribución de los datos fue normal, los resultados no representan un amplio espectro de la población en estudio, debiendo interpretarse los resultados con cautela. La falta de un grupo control, también dificulta la interpretación de resultados, ya que los cambios fueron determinados en un grupo autocontrol, por lo que queda abierta la posibilidad de considerar esto en futuros estudios.

El tiempo de duración del entrenamiento es otro aspecto a considerar, ya que la intervención duró 10 días, sin embargo, existe literatura que señala que un HIIT de bajo volumen, genera adaptaciones fisiológicas en seis sesiones o dos semanas de entrenamiento, no obstante, estos datos fueron obtenidos en población sana, lo que induce a investigar que sucede en sujetos con trastornos metabólicos ${ }^{49}$.

\section{Conclusión}

Los resultados demostraron cambios estadísticamente significativos en el tau rápido de la cinética off del $\mathrm{VO}_{2}$ luego de realizar un entrenamiento interválico de alta intensidad $(5 \times 55 \times 5)$ en un periodo de 10 días. Además, de una tendencia del resto de los parámetros hacia valores esperables, aunque sin lograr diferencias significativas. Pese a esto, se confirma la hipótesis planteada, lo cual podría sugerir que este tipo de entrenamiento, genera cambios positivos en sujetos con sobrepeso y obesidad, con respecto a la recuperación posterior al ejercicio físico. Sin embargo, se necesitan más estudios en el área para profundizar en los mecanismos fisiológicos relacionados, la incorporación de un grupo control y mayor número de muestras serían útiles para interpretar los resultados en forma más concluyente.

\section{Consideraciones éticas}

Todos los participantes firmaron el consentimiento informado. Los procedimientos fueron aprobados por el comité de ética de la Universidad Santo Tomás.

\section{Conflicto de interés}

Los autores expresan que no hay conflictos de intereses al redactar el manuscrito.

\section{Referencias}

1. Schlaich M, Straznicky N, Lambert E, Lambert G. Metabolic syndrome: a sympathetic disease?. Lancet Diabetes Endocrinol. 2015; 3(2): 148-157.

2. Organización Mundial de la Salud. Informe Mundial Sobre la Diabetes. Ginebra. 2016; (4): 1-4. 
3. Rodríguez DA, Garcia-Aymerich J, Valera JL, Sauleda J, Togores B, Galdiz JB, et al. Determinants of exercise capacity in obese and non-obese COPD patients. Respir Med. 2014; 108(5): 745-751.

4. Lambrick D, Faulkner J, Westrupp N, McNarry M. The influence of body weight on the pulmonary oxygen uptake kinetics in pre-pubertal children during moderate- and heavy intensity treadmill exercise. Eur J Appl Physiol. 2013; 113(8): 19471955.

5. Salome CM, King GG, Berend N. Physiology of obesity and effects on lung function. J Appl Physio. 2010; 108(1): 206-211.

6. Littleton SW. Impact of obesity on respiratory function. Respirology. 2012; 17(1): 43-49.

7. DeLorey DS, Wyrick BL, Babb TG. Mild-tomoderate obesity: implications for respiratory mechanics at rest and during exercise in young men. Int J Obes (Lond). 2005; 29(9): 1039-1047.

8. Kriketos AD, Baur LA, O'Connor J, Carey D, King $\mathrm{S}$, Caterson ID, et al. Muscle fibre type composition in infant and adult populations and relationships with obesity. Int J Obes Relat Metab Disord. 1997; 21(9): 796-801.

9. Barstow TJ. Characterization of VO2 kinetics during heavy exercise. Med Sci Sports Exerc. 1994; 26(11): 1327-1334.

10. Montero JC, Cúneo A, Facchini M, Bressan J. Comprehensive treatment of obesity and its prevention. An Sist Sanit Navar. 2002; 25(1): 175186.

11. Gómez S, Marcos A. A comprehensive approach to treating adolescent obesity. Rev Med Univ Navarra. 2006; 50(4): 23-25.

12. Corte de Araujo AC, Roschel H, Picanço AR, do Prado DM, Villares SM, de Sá Pinto AL, et al. Similar health benefits of endurance and highintensity interval training in obese children. PLoS One. 2012; 7(8): e42747.

13. Duarte C, de Castro C, de Araujo C. Treinamento para disfunção vagal cardíaca com repetições da transição repouso-exercício. Rev Bras Ativ Fis Saúde. 2013; 18(6): 688-967.

14. Bell C, Paterson DH, Kowalchuk JM, Padilla $\mathrm{J}$, Cunningham DA. Comparison of modelling techniques used to characterise oxygen Uptake kinetics During the on- transient of exercise. Exp Physiol. 2001; 86(5): 667-676.

15. Vivier, L. Évaluation de la contribution du système aérobie lors d'une épreuve de $1500 \mathrm{~m}$ en curse à pied. 2005. 122.

16. Hughson RL. Oxygen uptake kinetics: historical perspective and future directions. Appl Physiol Nutr
Metab. 2009; 34(5): 840-850.

17. Lupton H, Hill A. An analysis of the effects of speed on the mechanical efficiency of human muscular movement. J Physiol. 1923; 57(6): 337-353.

18. Hill A, Long C, Lupton H. Muscular exercise, lactic acid, and the supply and utilisation of oxygen. Proceedings of the Royal Society of London. Series B, containing papers of a biological character. 1924; 97(681): 84-138.

19. Gaesser GA, Brooks GA. Metabolic bases of excess post-exercise oxygen consumption: a review. Med Sci Sports Exerc. 1984; 16(1): 29-43.

20. McArdle W, Katch V, Katch F; Schofield D. Fisiología del ejercicio: energía, nutrición y rendimiento humano. 1a edición. Madrid: Alianza: Consejo Superior de Deportes, 1995; 2: 119-136.

21. Stupnicki R, Gabryś T, Szmatlan-Gabryś U, Tomaszewski P. Fitting a single-phase model to the post-exercise changes in heart rate and oxygen uptake. Physiol Res. 2010; 59(3): 357-362.

22. Matsuura C, Meirelles C, Gomes P. Gasto energético e consumo de oxigênio pós-exercício contraresistência. Rev Nutr. 2006; 19(6): 729-740.

23. Neto A, Farinatti P. Consumo de oxigênio após exercício resistido: uma abordagem crítica sobre os fatores determinantes de sua magnitude e duração. Braz. J Biomotricity. 2009; 3(2): 96-110.

24. Junior N. Matemática da cinética do VO2 e da contribuição do sistema de energia durante o exercício: um estudo de revisão. Rev Brasil Prescrição Fisiol Exercício. 2012; 6(36): 578-603.

25. Álvarez GE, Beske SD, Ballard TP, Davy KP. Sympathetic neural activation in visceral obesity. Circulation. 2002; 106 (20): 2533-2536.

26. Espinoza A, Acuña S, Sánchez P, Zafra E. Revisión bibliográfica: Efectos del entrenamiento interválico de alta intensidad en el balance autonómico y la cinética del consumo de oxígeno en sujetos obesos. Rev Horiz Cienc Act Fís. 2016; (7): 30-45.

27. Gibala MJ, Little JP, Macdonald MJ, Hawley JA. Physiological adaptations to low -volume, highintensity interval training in health and disease. J Physiol. 2012; 590: 1077-1084. DOI: 10.1113/ jphysiol.2011.224725.

28. Álvarez LC, Ramírez-Campillo R, Flores OM, Henríquez-Olguín C, Campos JC, Carrasco V, et al. Respuestas metabólicas inducidas por ejercicio físico de alta intensidad en mujeres sedentarias con glicemia basal alterada e hipercolesterolemia. Rev Méd Chile. 2013; 141(10): 1293-1299.

29. Gibala MJ, Little JP, Macdonald MJ, Hawley JA. Physiological adaptations to low-volume, highintensity interval training in health and disease. $\mathrm{J}$ 
Physiol. 2012; 590(5): 1077-1084.

30. Hernández R, Fernández C, Baptista P. Metodología de la investigación. 5ta edición. México: McGrawHill Interamericana, 2010.

31. Jones A, Poole D. Oxygen Uptake kinetics in sport, exercise and medicine. J Sports Sci Med. 2005; 4(1): 84.

32. Rosso S. La Cinética del Consumo de Oxígeno. Eur J Appl Physiol. 2013; (72): 37-43.

33. Forman DE, Myers J, Lavie CJ, Guazzi M, Celli B, Arena R. Cardiopulmonary exercise testing: relevant but underused. Postgrad Med. 2010; 122(6): 68-86.

34. Salvadego D, Lazzer S, Busti C, Galli R, Agosti F, Lafortuna $\mathrm{C}$, et al. Gas exchange kinetics in obese adolescents. Inferences on exercise tolerance and prescription. Am J Physiol Regul Integr Comp Physiol. 2010; 299(5): R1298-1305.

35. Paterson DH, Whipp BJ. Asymmetries of oxygen uptake transients at the on-and offset of heavy exercise in humans. J Physiol. 1991; 443: 575-586.

36. Padilla-Pérez, J. The on- and off-transient phase two VO2 kinetics during submaximal exercise in young men. Rev Hosp Jua Mex 2013; 80 (1): 6-13.

37. Barstow TJ, Molé PA. Linear and nonlinear characteristics of oxygen uptake kinetics during heavy exercise. J Appl Physiol. 1991; 71(6): 2099-2106.

38. Özyener F, Rossiter HB, Ward SA, Whipp BJ. Influence of exercise intensity on the on- and offtransient kinetics of pulmonary oxygen uptake in humans. J Physiol. 2001; 533(Pt 3): 891-902.

39. Engelen M, Porszasz J, Riley M, Wasserman K, Maehara K, Barstow TJ. Effects of hypoxic hypoxia on $\mathrm{O} 2$ uptake and heart rate kinetics during heavy exercise. J Appl Physiol. 1996; 81(6): 2500-2508.

40. Marwood S, Roche D, Garrard M, Unnithan VB. Pulmonary oxygen uptake and muscle deoxygenation kinetics during recovery in trained and untrained male adolescents. Eur J Appl Physiol. 2011; 111(11): 2775-2784.

41. Fukuoka Y, Grassi B, Conti M, Guiducci D, Sutti M, Marconi C, et al. Early effects of exercise training on on-and off-kinetics in 50-year-old subjects. Pflugers Arch. 2002; 443(5-6): 690-697.

42. Billat VL, Mille-Hamard L, Demarle A, Koralsztein JP. Effect of training in humans on off-and ontransient oxygen uptake kinetics after severe exhausting intensity runs. Eur J Appl Physiol. 2002; 87(6): 496-505.

43. Sousa A, Rodríguez FA, Machado L, Vilas-Boas JP, Fernandes RJ. Exercise modality effect on oxygen uptake off- transient kinetics at maximal oxygen uptake intensity. Exp Physiol. 2015;
100(6): 719-729.

44. López-Chicharro J, Fernández-Vaquero A. Fisiología del Ejercicio. 3era edición. Madrid: Ed. Médica Panamericana. 2006.

45. Hernández-Jiménez, S. Fisiopatología de la obesidad. Gac Méd Méx. 2004; 140(2): 27-32.

46. Heath, Edward M. Exercise Physiology: Energy, Nutrition, and Human Performance. Medicine \& Science in Sports \& Exercise. 2002; 34: 903.

47. Boyd JC, Simpson CA, Jung ME, Gurd BJ. Reducing the intensity and volume of interval training diminishes cardiovascular adaptation but not mitochondrial biogenesis in overweight/obese men. PLoS One. 2013; 8(7): e68091.

48. McNarry MA, Lambrick D, Westrupp N, Faulkner J. The influence of a six-week, high-intensity games intervention on the pulmonary oxygen uptake kinetics in prepubertal obese and normal-weight children. Appl Physiol Nutr Metab. 2015; 40(10): 1012-1018.

49. Salassi J. The acute effects of various highintensity interval training (HIIT) protocols on cardiopulmonary and metabolic function. California State University, Long Beach. ProQuest Dissertations Publishing, 2014.

50. Liang H1, Ward WF. PGC-1 $\alpha$ : a key regulator of energy metabolism. Adv Physiol Educ. 2006; 30(4): 145-151. 